\title{
Interactive Computer Modules for \\ Undergraduate Chemical Engineering Instruction
}

\author{
H. Scott Fogler, Susan M. Montgomery, and Robert P. Zipp* \\ Chemical Engineering Department, University of Michigan, Ann Arbor, Michigan 48109-2136
}

\begin{abstract}
Interactive computer modules have been developed for four of the core courses in the Chemical Engineering curriculum: Introduction to Chemical Engineering, Fluids/Transport, Separations, and Kinetics. These modules generally consist of a review of the material, followed by an interactive problem-solving session, which may include a computer simulation of the processes involved. The problem is often presented as part of a scenario, to capture the student's interest, and hints are available to guide the student. This study examines the components of these modules, as well as considerations that educators should take into account when developing interactive computer modules. (c) 1992 John Wiley \& Sons, Inc.
\end{abstract}

\section{INTRODUCTION}

The University of Michigan's Chemical Engineering Department has long been an innovator in the use of computers to enhance chemical engineering education. One component of the thrust toward computer-aided instruction has been the development of IBM-PC-based stand-alone modules that cover the main concepts in most of the core courses in the Chemical Engineering curriculum. The computer modules take advantage of both the interactive nature of the computer and its computational capabilities, as well as bringing examples from emerging technologies into the curriculum.

Interactive computer modules allow the student to review and demonstrate mastery of the material

\footnotetext{
* Current address: The Upjohn Co., Kalamazoo, Michigan. Computer Applications in Engineering Education, Vol. 1(1) 1124 (September/October 1992)

(C) 1992 John Wiley \& Sons, Inc. CCC 1060-3773/92/010011-14
}

at his / her own pace, and they provide immediate feedback to a student's responses. The more advanced modules allow students to explore the effects of parameter variations on a system, perform simulations to optimize its performance, and carry out simulated experiments from which they can obtain the data needed to model the system.

We have developed over two dozen modules that span the introductory, fluids and transport, separations, and reaction kinetics courses. Some of the modules developed or being tested have been distributed to other universities, where the response has been very enthusiastic. This article focuses on the progress that has been made on both the computer and pedagogical aspects of module development, and on our plans for the future.

\section{PROJECT HISTORY}

Kulik and Kulik, both of the Center for Research on Learning and Teaching (CRLT) at the University 
Table 1 Primary Components of Interactive Computer Modules

- Introduction

- Review of pertinent fundamentals

- Demonstration

- Interactive exercise

- A branching component

- A solution to the exercise

- Evaluation

of Michigan, reported that most studies found that computer-based instruction had positive effects on the students[1,2]. In the early 1970s, one of the authors (Fogler) developed a number of modules on reaction engineering for use on the University of Michigan's mainframe time-sharing computer. When these simulations were demonstrated at a national meeting, they were enthusiastically received by chemical engineering faculty at other colleges and universities, several of whom requested copies of the programs. Unfortunately, these interactive programs were not easily transportable to computing environments at other universities. In particular, idiosyncrasies in the local graphics-display software caused much of the transportability problem, although operating-system incompatibilities also played a role. However, in response to the rapid evolution of microcomputer-based work stations, many universities have undertaken major microcomputer acquisitions for their students, and today, students in virtually every chemical engineering de- partment in the U.S. and Canada have access to a large number of IBM (or IBM-compatible) personal computers. In the early 1980 s, many of the original reaction engineering modules were translated to an IBM format, using BASICA. In 1988 we received funds as part of the National Science Foundation grant "A Focus on Developing Innovative Engineers" to develop a new generation of interactive computer modules for chemical engineering.

\section{Choice of Authoring System}

Several authoring systems were evaluated by a CACHE Corporation (Computer Aids for Chemical Engineering, Austin, TX) task force. The authoring system of choice would allow high-resolution color graphics, distributable utility subroutines, easy portability between graphics boards, support of a mathematics coprocessor, and result in a decrease in the time spent preparing modules. Additional criteria included: the ability to mix text and graphics, good computational facilities for animation and numerical calculation work, optional device drivers, access to DOS within the system, and reasonable licensing fees or no royalties. Tests were performed which included programming language and calculation speed tests, software support tests, graphics system tests, and lesson-editing tests. The authoring language, UNISON (Courseware Applications, Inc., Champaign, IL 61820), was found to satisfy the above criteria.

As drug patches are something new to you, you wisely decide to learn more about them Your research yields the following information:

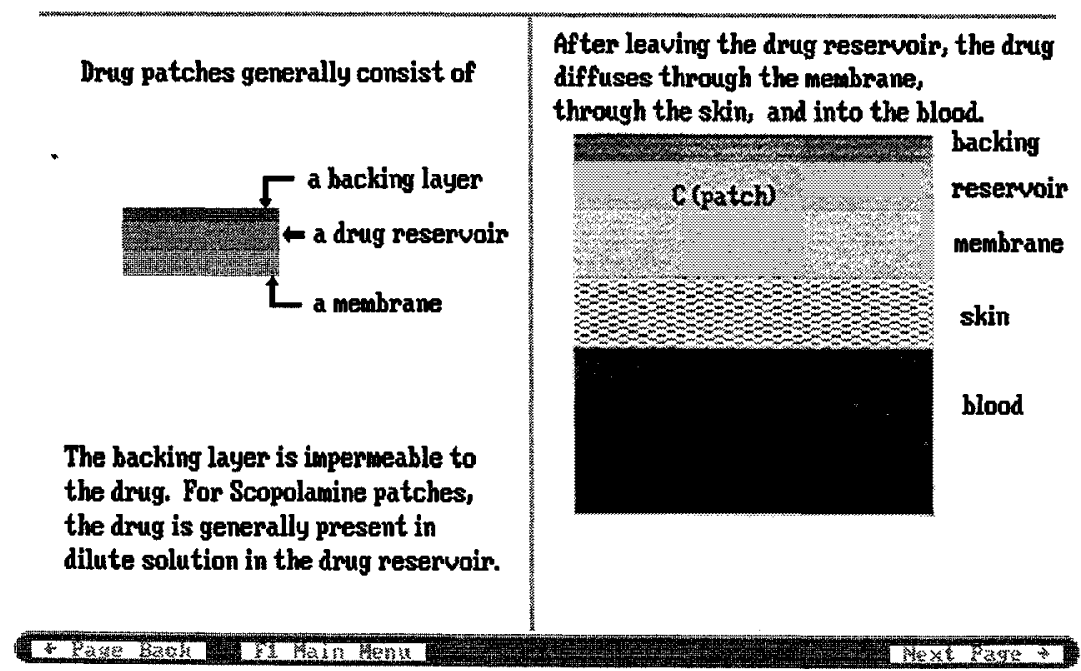

Figure 1 Introduction Screen, "Drug Patch," Diffusion Module. 
MASS TRANSPORT: FICK'S LAW (continued)

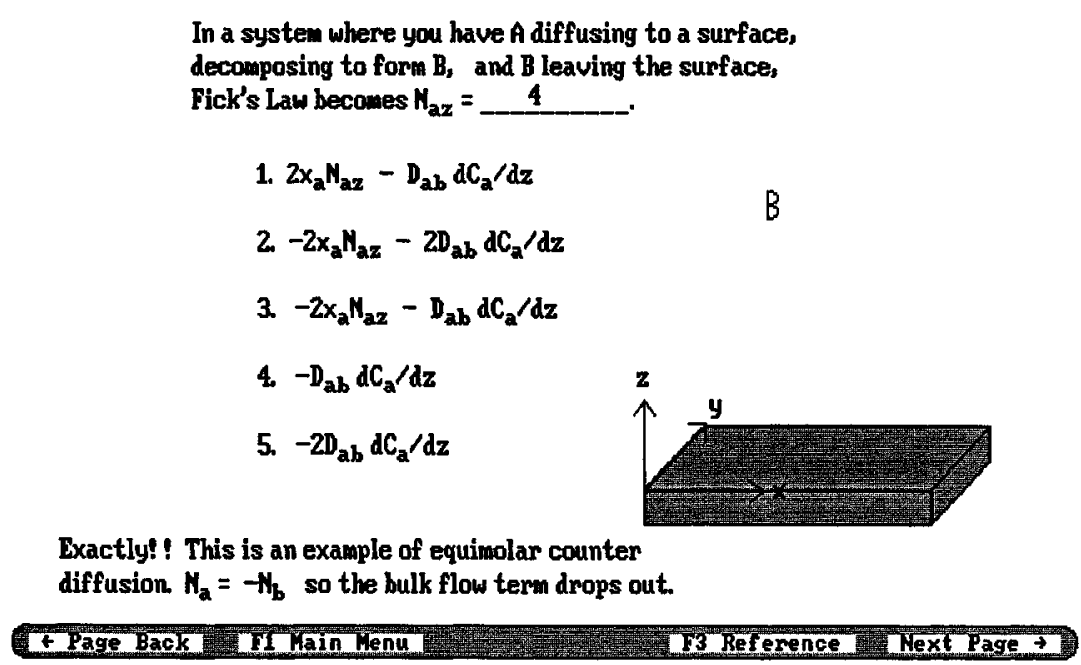

Figure 2 Review Screen, "Drug Patch," Diffusion Module.

UNISON was used until 1990 to develop the modules in the introductory chemical engineering course, as well as some in the fluids course. After a new evaluation by one of the authors (Zipp), the QUEST authoring system (Allen Communication, Inc., Salt Lake City, UT) was chosen. In addition to the many positive features of UNISON, QUEST includes an interactive nonprogramming-based authoring and graphics system that has greatly decreased the module development time. In addition, a PASCAL-like language is available, and may be used to incorporate complex calculation and simulation programs into the module. By using an IBMPC-based authoring system to develop the modules, the development time and portability problem, so formidable a few years ago, have now been largely eliminated.

\section{Current Module Development and Distribution Protocol}

Through the cooperation of faculty members, postdoctoral fellows and undergraduate students, we have been able to develop a large number of interactive computer modules. The undergraduate students' previous experience with computer modules in their courses provided much insight into ways of making the modules more interesting, relevant, and helpful to other students. Undergraduate students are hired during the summer as the initial programmers for each of the modules, under the supervision of postdoctoral fellows. Faculty members within the department as well as from other universities serve as technical advisors, conferring with the students at every stage of module development to ensure a high level of accuracy of the material and effectiveness of its presentation.

Once the preliminary version of a set of modules is complete, they are tested first with students in our department, using the network system available in the College of Engineering. Some modules have also been distributed to volunteer faculty testers at other universities, and have been demonstrated at the national meeting of the American Institute of Chemical Engineers. In addition, this summer there will be a workshop at the American Society for Engineering Education's summer school for chemical engineering faculty, held every five years. This workshop will focus on the materials developed and how best to incorporate them into a course. After appropriate revision and clarification of the materials, the modules are ready for distribution. At the completion of the development phase of the project the modules will be distributed by the CACHE Corporation.

\section{MODULE COMPONENTS}

After discussions with researchers from CRLT on how to best reinforce the material and foster independent learning, we designed the modules to include the primary components as shown in Table 1 . 


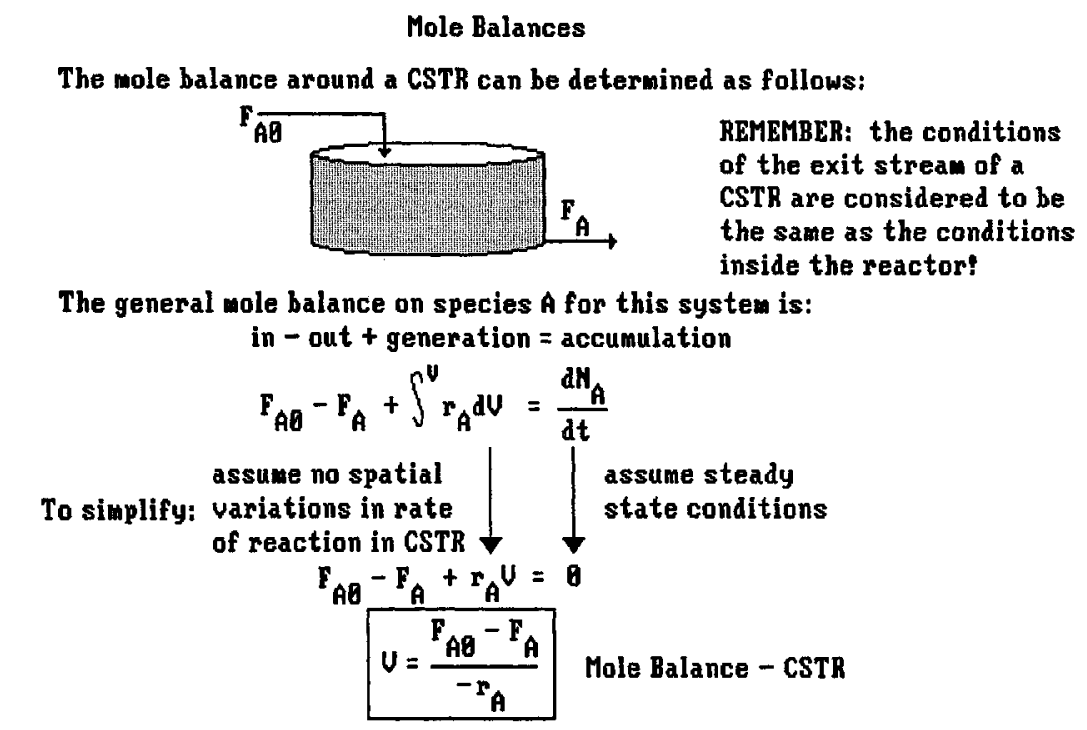

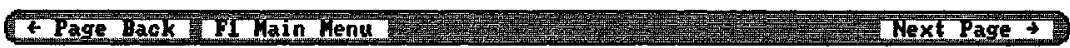

Figure 3 Review Screen, "Reactor Staging" Module.

The introduction presents the student with the major concept covered by the module and/or the process to be examined. Figure 1 shows an example from a module on diffusion that focuses on the production of drug patches. The review section allows the student to re-examine the material presented in class lectures at their own pace, often with feedback, as in Figure 2. In addition, the use of graphics during manipulation of equations in this section, as shown in Figure 3, from the reactor staging module, serves as a pedagogical aid that is very hard to duplicate in the classroom. A student with a good understanding of the material may omit the review section, and proceed to the problem-solving portion of the module. Other students benefit from an additional presentation of the key concept.

The interactive exercise is the primary component of the module. It is preceded by a short dem-

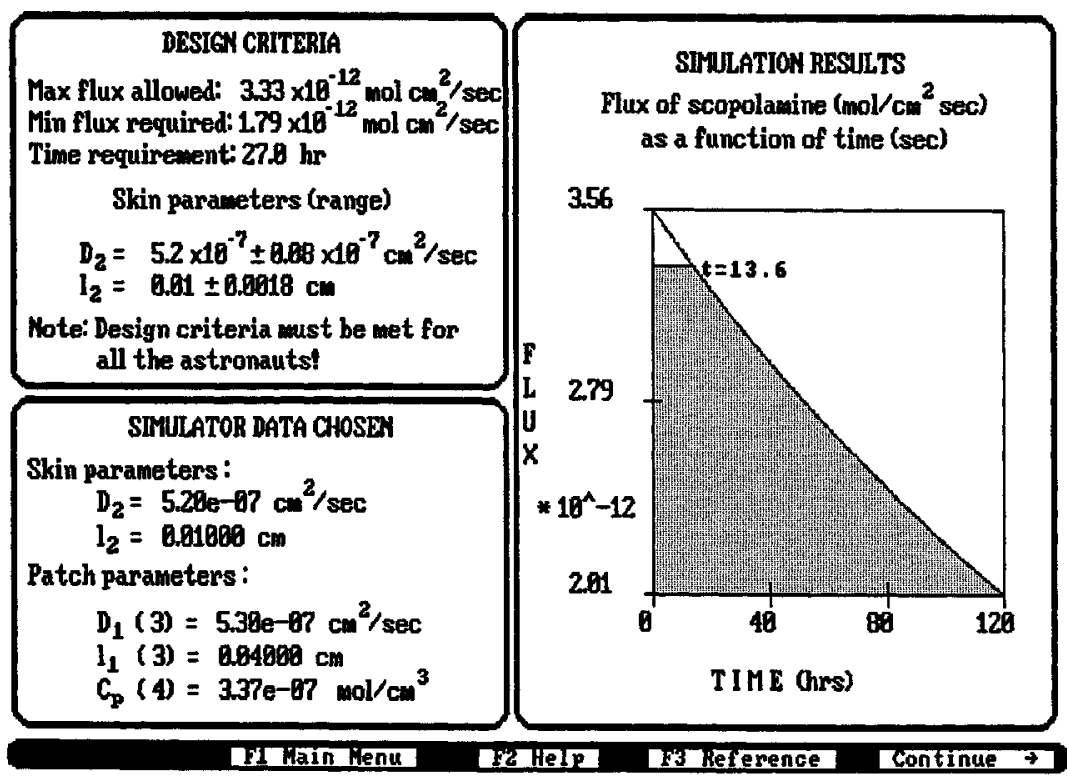

Figure 4 Simulation Screen, “Drug Patch,” Diffusion Module. 


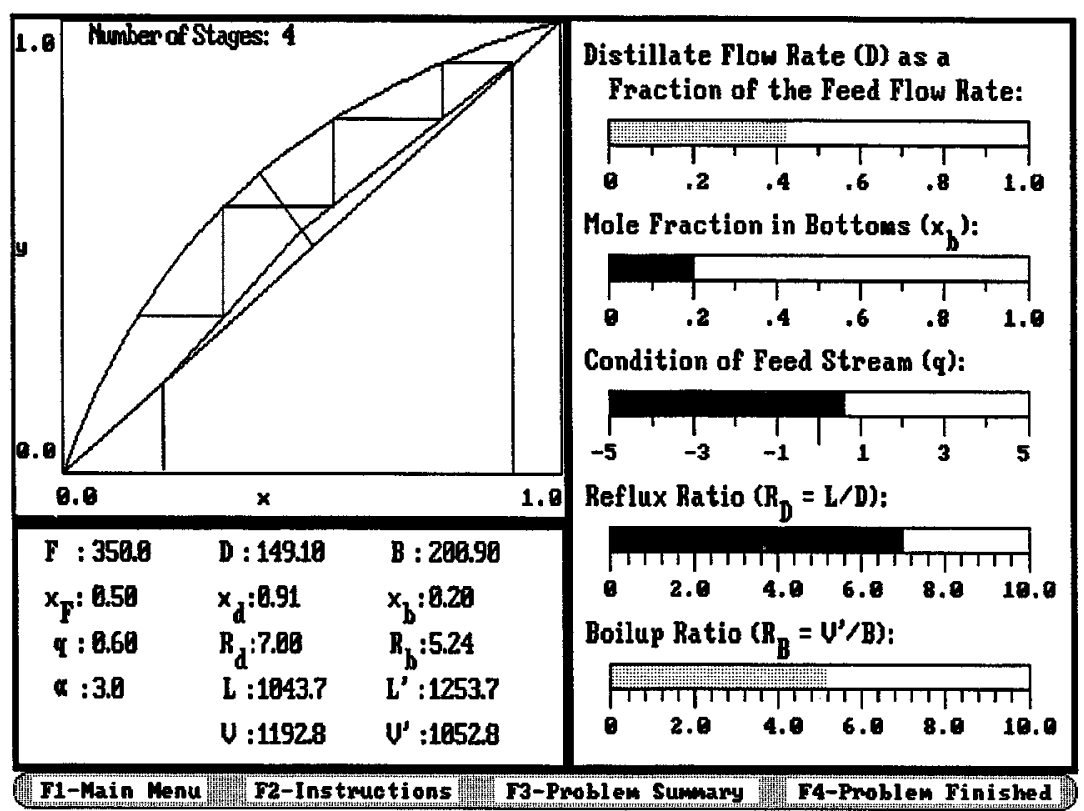

Figure 5 Simulation Screen, "McCabe-Thiele," Distillation Module.

onstration that familiarizes the student with its key aspects. In some of the modules that cover introductory material this section is an interactive testing section, rather than an exploration section. Modules on more advanced concepts often consist of simulations of processes. These modules can provide for the planning of experiments by allowing the students to choose an experimental system, to take simulated "real" data, to modify experiments to obtain data in different parameter ranges, and to design a piece of equipment or process. The student can focus on applying the concepts in a design mode, rather than spending a large amount of time simply generating the data from the given equations, with no clear understanding of the underlying concepts. Figure 4 shows the results of a simulation in the drug-patch

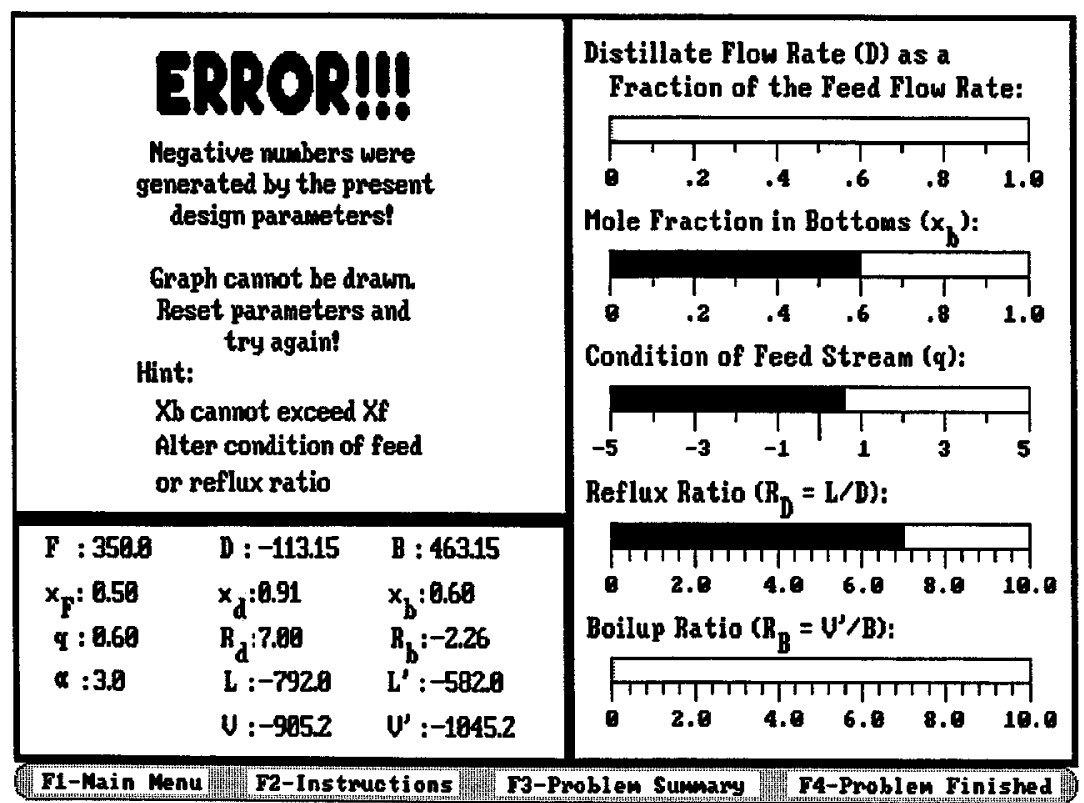

Figure 6 Simulation Screen with Warning, "McCabe-Thiele" Distillation Module. 
module described above. Modules also allow the student to analyze the effect of various process parameters. In the module on distillation using the McCabe-Thiele method, for example, the student may vary a number of parameters, as shown in Figure 5. One clear advantage of using a computer is the availability of immediate guidance based on a student's decisions, which helps to correct any conceptual errors as soon as they are made, increasing the chances that the material will be retained. Should the student enter a physically impossible set of parameters for the distillation, a warning, shown in Figure 6 , is issued, along with suggestions for correcting the error.

Other simulations are more academic, such as the nonisothermal reactor simulation, shown in Figure 7. This simulation can be used either as a stand-alone module, in which the student learns the effect of each parameter in the process of fulfilling a given design goal, or it can be used in the independent study project mode, in which the professor might ask specific questions, such as: "What is the effect of increasing the heat exchanger area on the temperature and conversion profiles down a PFR with an exothermic reaction?" That question can be answered after a brief exploration using the simulator. This type of simulation could also be used in the classroom with the aid of a monitor-to-screen projector to ask "What if . . . ?" questions of the class. The students could suggest expected results, and compare their predictions to the results obtained from the simulation.
Table 2 Considerations in Developing Computer Modules

- Ease of use

- Introduction of new technologies

- Maintaining the focus on the concepts

- No tediousness!

- Promoting learning

- Individual guidance

After the interactive portion, the student's performance is evaluated, and a score, encoded in a performance number, is generated.

\section{WHAT WE HAVE LEARNED}

A significant portion of our efforts has gone to the development of a pedagogical philosophy that makes use of the computer's graphic, interactive, and computational capabilities. In addition, the sophistication required to satisfy a more computer-oriented student body has resulted in the need for more "polished" modules than in the past. The resulting interactive computer module should be a learning experience that supplements the typical classroom and standard homework activities.

There are many advantages to using computerbased learning tools. There are also some pitfalls that one must be aware of and avoid. In addition

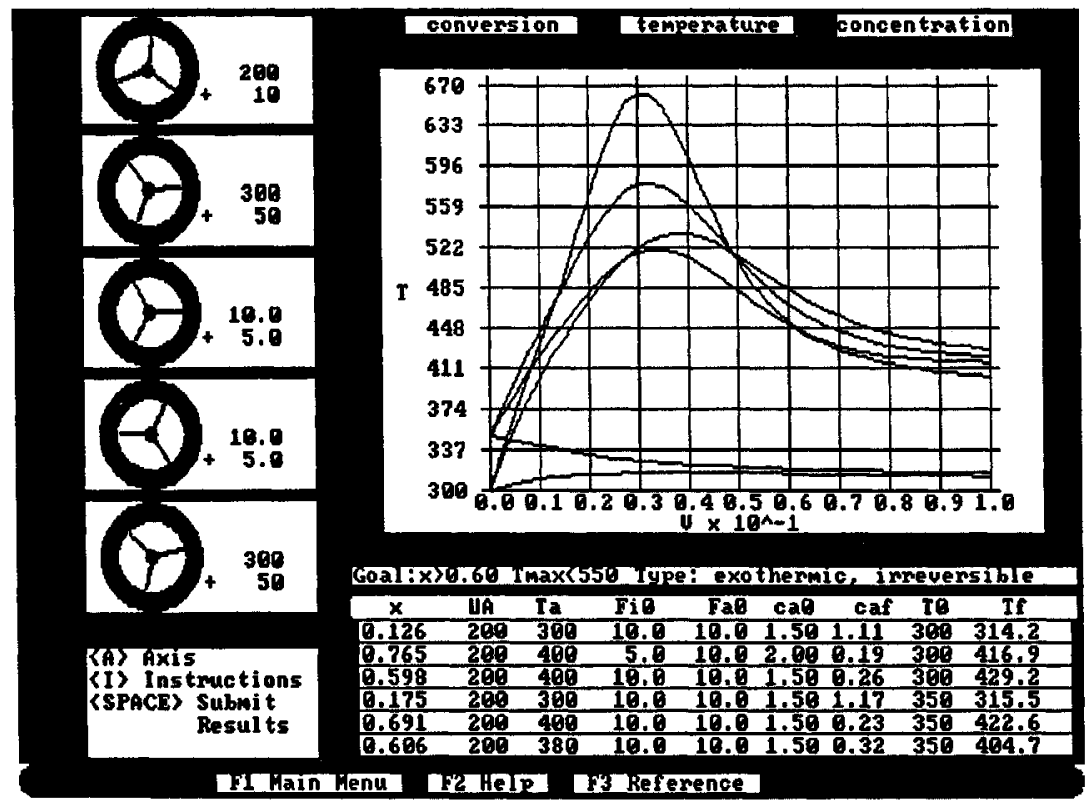

Figure 7 Simulation Screen, "Nonisothermal Reactors" Module. 
When you reach the island, you realize that to survive, you must find some drinking water. A quick search of the island reveals only one pond of water. However, you suspect that this pond may contain some toxic substances as well.

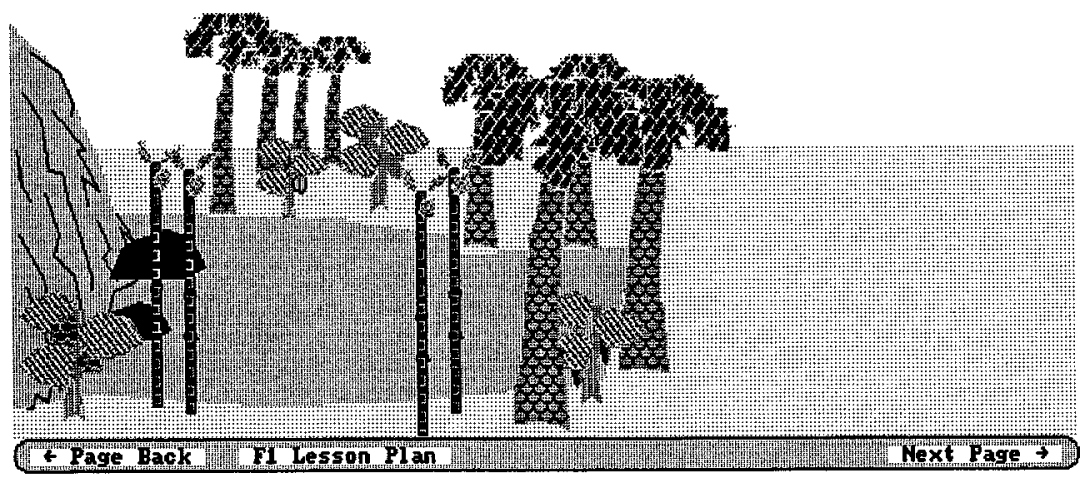

Figure 8 Introduction Screen, "Rescue," Adsorption Module.

to ensuring the technical accuracy of the material and simulations in the module, there are other considerations we have become aware of through our testing and the comments of the external faculty testers. Some of the aspects of the use of interactive computer modules in engineering education that should be considered by all computer module developers are summarized in Table 2.

\section{Ease of Use}

The module interface must be easy to use and consistent from module to module-so it does not in- terfere with the student's learning. A user interface that remains consistent throughout the modules has been developed. All the modules include a command bar at the bottom of the screen, shown in many of the figures above, that presents a consistent set of options to the students. The keys used to activate these options, which include exiting to the module's main menu, activating a hint screen, a reference screen, or a calculator, remain the same from module to module. The reference screen contains such information as conversion factors, measured results, or necessary equations. The pop-up

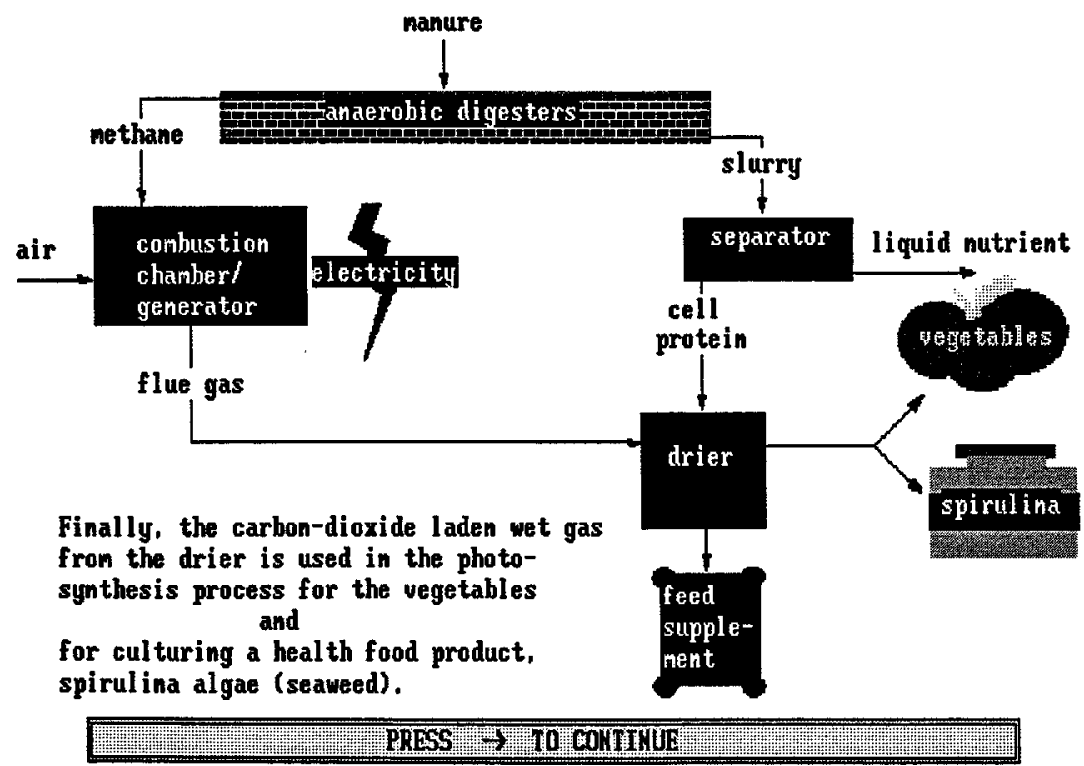

Figure 9 Introduction Screen, "Hawaii," Material Balances Module. 
THE PROCESS FLOHSHEET

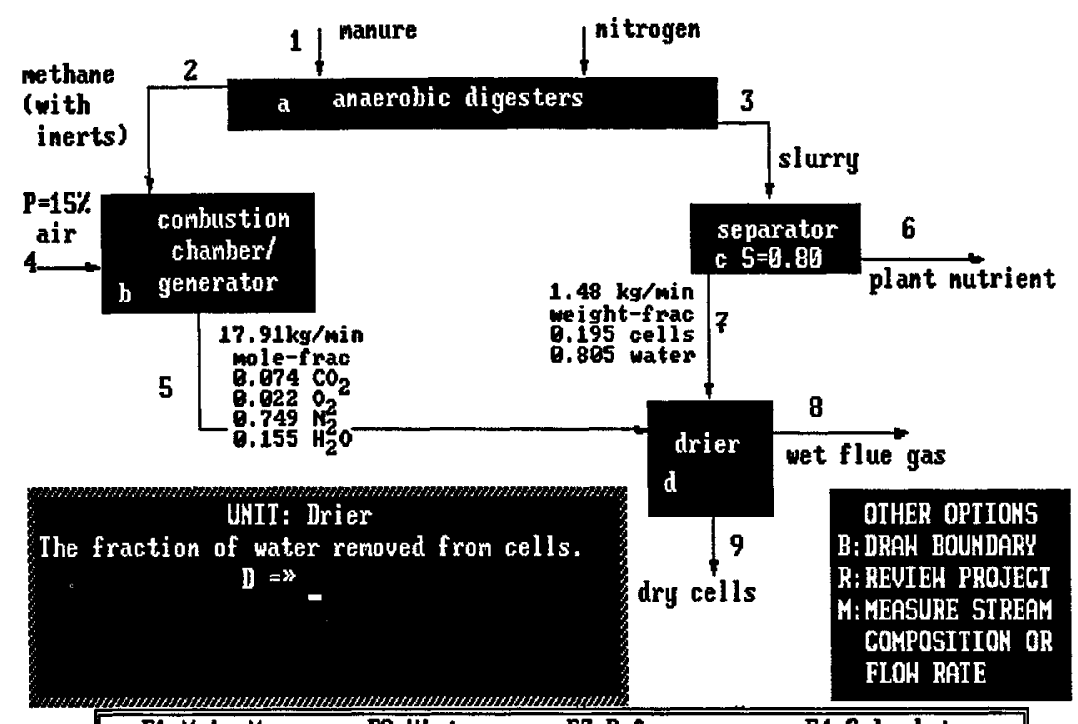

F1: Kain Menu F2:Hint F3:Reference F4:Calculator

Figure 10 Interaction Screen, "Hawaii," Material Balances Module.

calculator used is the POLYMATH calculator. A full screen tutorial is available at the beginning of the unit conversion module; a condensed tutorial is available in all other modules that use the calculator. We also often incorporate students' comments on other interface aspects of the modules, to ensure that a student's understanding of the material is not hindered by the interface.

\section{Introduction of New Technologies / Focus on Concepts}

The interactive computer modules are an ideal medium to introduce the student to applications of the course concepts, particularly to new technologies, such as in the drug-patch module discussed earlier. A second example, in a module on adsorption, in-

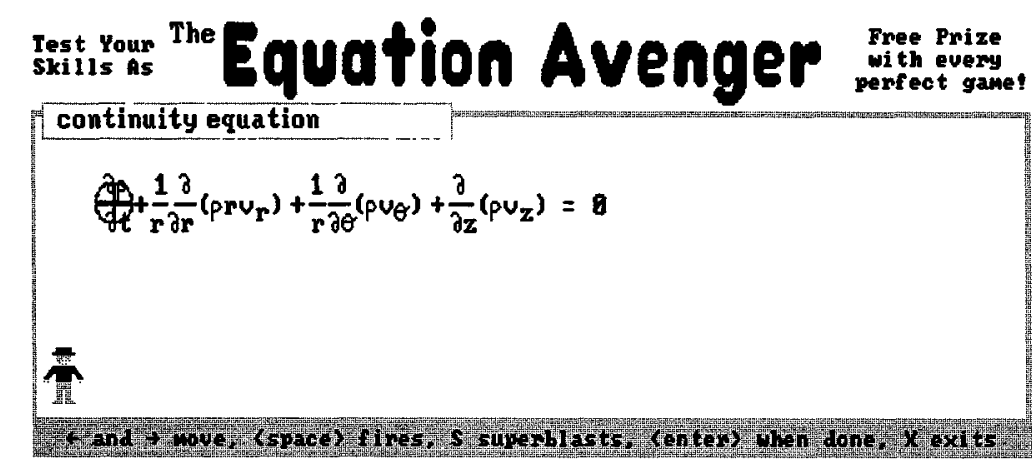

Problew number 1 :

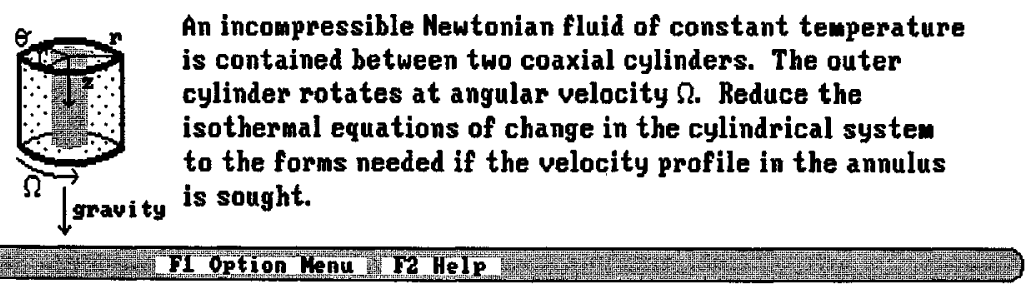

Figure 11 Interaction Screen, "Shoot," Transport Equations of Change Module. 


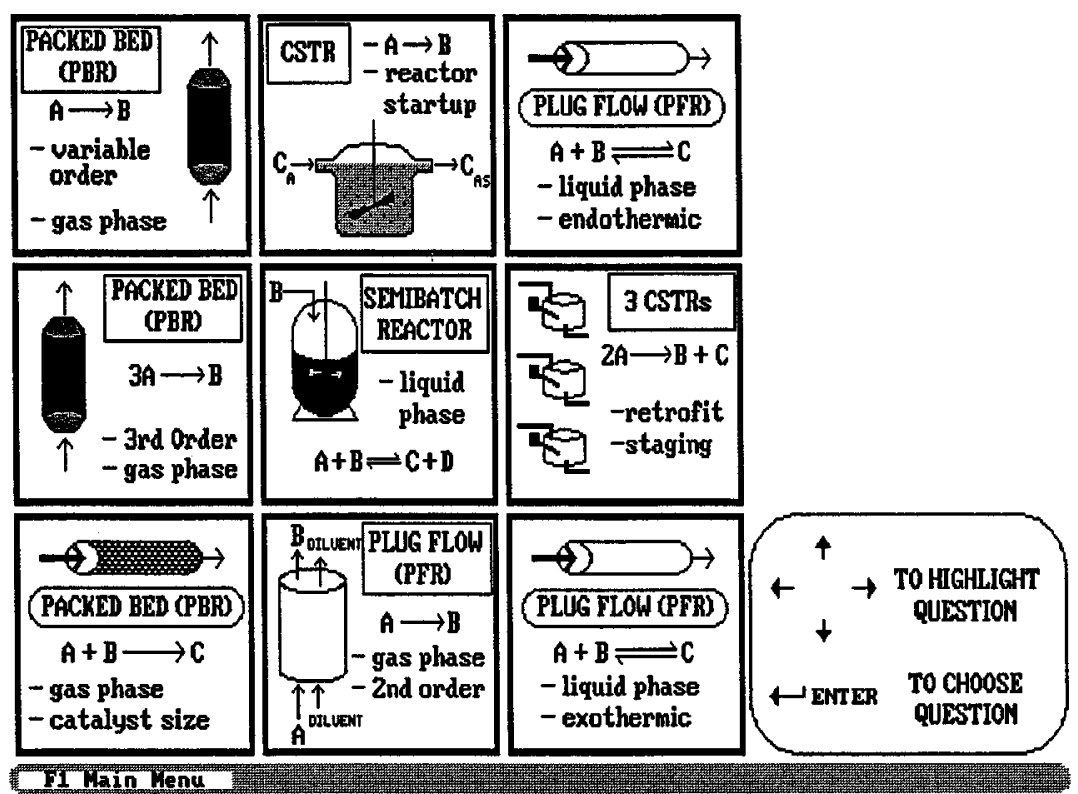

Figure 12 Simulation Selection Screen, “Tic-Tac-Toe," Isothermal Reactors Module.

volves a scenario in which the student is purportedly stranded on a desert island, and must find a way to purify the water. A sample screen from this module is shown in Figure 8. The student can make use of a simulator in which the effectiveness of various combinations of type of bamboo (column) and bean species (adsorptive material) can be compared. Seeing these applications should increase the student's interest in understanding the concepts pre- sented in class. In addition, the student can explore the effect of parameter changes on the system and gain an intuitive feeling for the process, rather than simply memorizing equations. These applications have even been introduced in the introductory course. In the mass balances module the student is first introduced to an actual biowaste facility (Figure 9 ), then must decide which process data to request in order to determine the value of certain key process

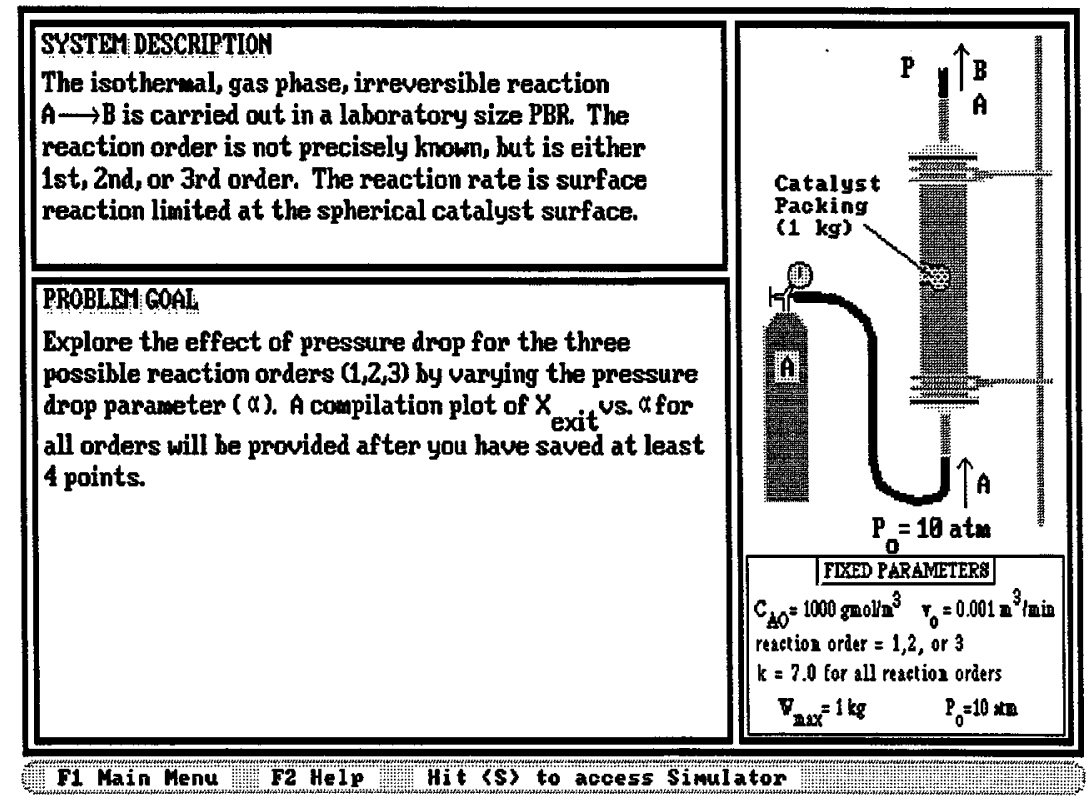

Figure 13 Introduction to a Simulation, "Tic-Tac-Toe," Isothermal Reactors Module. 


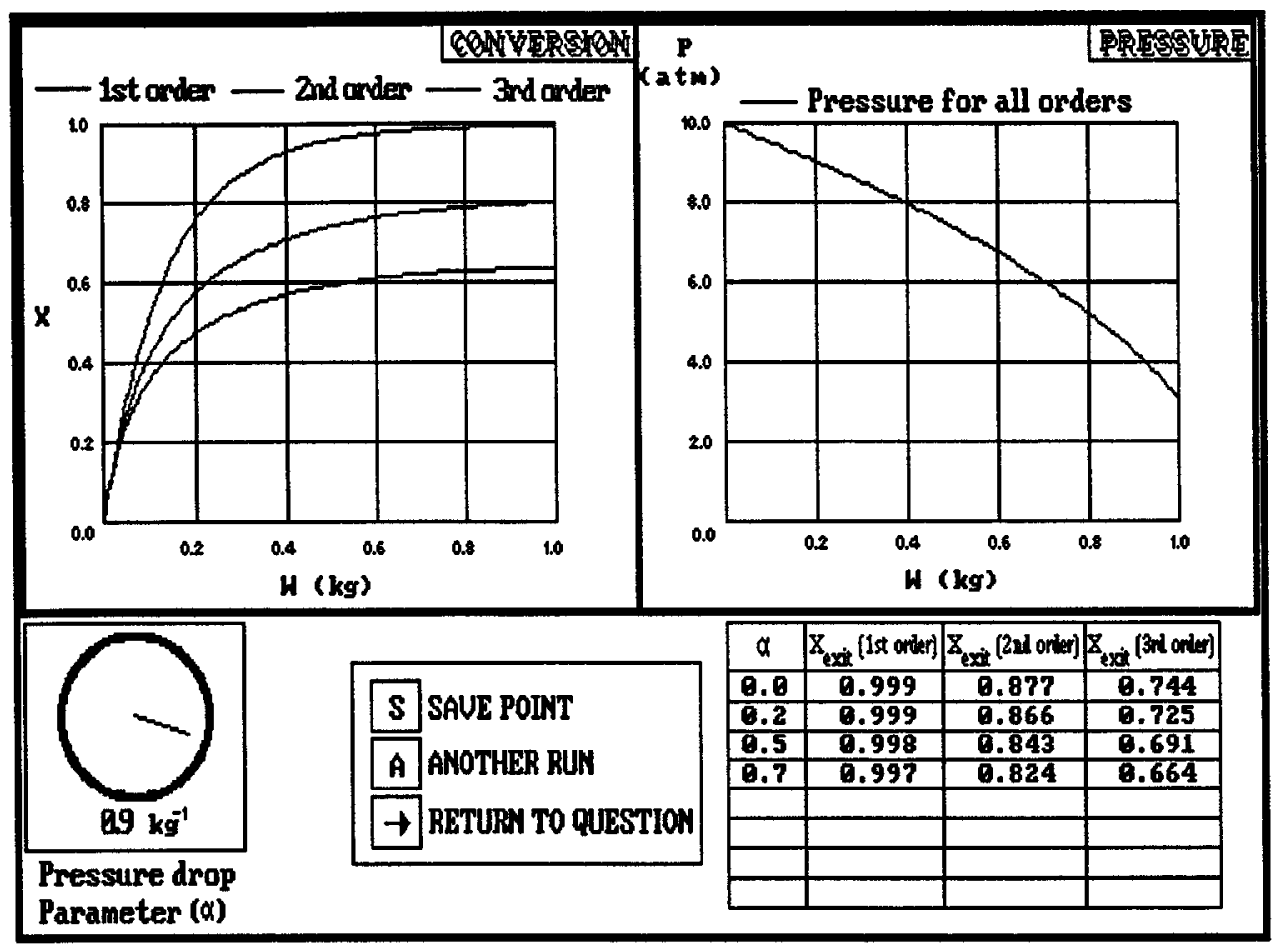

Figure 14 Simulation Screen, "Tic-Tac-Toe," Isothermal Reactors Module.

parameters for the system, using the process flowsheet shown in the interaction screen (Figure 10).

\section{No Tediousness}

We have used many approaches to keep the student's attention when running the modules. A gaming aspect is introduced into some of the modules, such as the "shooting gallery" transport module, in which the student must simplify the equations of change for a particular momentum or energy balance problem. This is done by eliminating the terms of the equations that are equal to zero, in an analogy to an amusement park's shooting gallery, as shown in Figure 11. The gaming aspect is not meant to trivialize the concepts involved, but simply to prevent the process from becoming tedious. Another aspect of this issue is ensuring that students who already understand the concepts do not spend inordinate amounts of time demonstrating this understanding. At the same time, those students who need the additional exposure should obtain it. The isothermal reactor design module achieves this by presenting the student with nine individual simulations, covering the effects of various parameters, in a tic-tac-toe pattern, as shown in Figure 12. The student must successfully master the concepts in enough squares to complete the tic-tac-toe. Students with a clear understanding of the material need only look at three of the squares, while students who are not able to show this understanding in the first three squares have additional opportunities to explore and learn using the other simulations.

\section{Promoting Learning}

The concept of allowing students to explore the effect of system parameters by providing them with unlimited options seems at first to have many benefits. One major drawback, however, is the temptation for the students to "twiddle the knobs" until the desired result occurs, with no clear understanding of the parameter effects and, in some cases, without even clear knowledge of which parameters were varied! To increase the amount of learning that occurs during these computer-simulation sessions, the number of parameters that a student may choose is sometimes limited, as in the McCabe-Thiele module described earlier, in which only three of the five parameters may be varied during a given session. In the Tic-Tac-Toe module, dividing the module into nine individual simulations allows the students to explore the effect of one parameter at a time. After choosing a simulation, the student is given a 


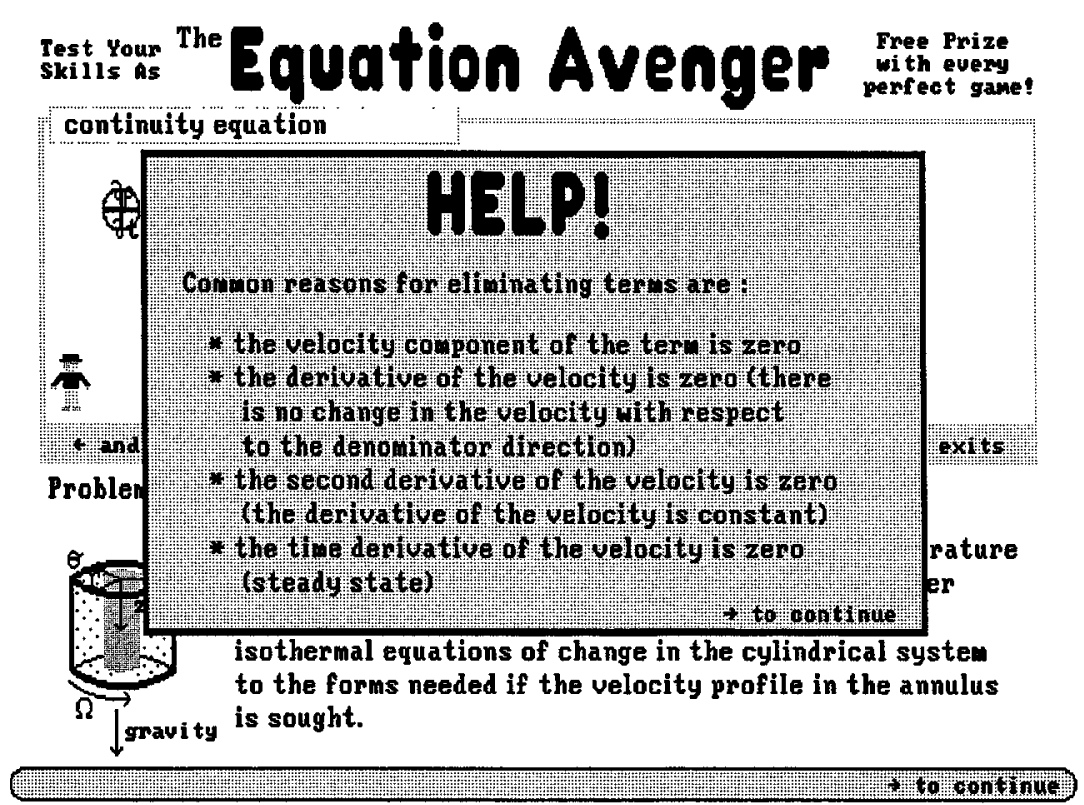

Figure 15 Interaction Screen with Hints, "Shoot," Transport Equations of Change Module.

brief description of the process, such as the one shown in Figure 13. The student must use the simulator, shown in Figure 14, to examine the effect of the given parameter on the reactor performance. Then the student must correctly answer two out of three follow-up questions to demonstrate an understanding of the processes that lead to the observed results. Another possible method to prevent random varying of the parameters to achieve a certain goal is to assign a set of questions that address the effect of specific parameters, to be answered using the simulation in the module. This method was mentioned earlier in the discussion of the nonisothermal reactors module, whose simulation screen was shown in Figure 7.

\section{Individual Guidance}

An advantage of the computer modules over standard homework exercises is the individual guidance provided to the student, which can only be duplicated with one-on-one mentoring. An example of guidance provided when a student makes mistakes was shown in the discussion of the McCabe-Thiele module (Figures 5 and 6 ). In addition, suggestions are often available to students who are "stumped" and need ideas on how to proceed. The student need only press the F2 "hint" key. For example, a student reviewing simplification of the equations of change using the "Shooting Gallery" module discussed ear- lier (Figure 11), may not have a good intuitive feeling for when terms would be equal to zero. A request for assistance would produce the hint screen shown in Figure 15. Finally, in those modules that do involve multiple choice questions, the module responds to an individual's choice by explaining the reasons that choice is correct or incorrect, so that the student can clarify any misconceptions immediately.

One additional aspect that we had not expected to observe should be noted. In earlier modules the student was presented with a score, along with a coded performance number to be submitted for grading purposes. Discussions with undergraduate students revealed that, in an attempt to obtain the highest possible score, some students would resort to deciphering the performance number, and/or running the module an inordinate number of times. Clearly, the additional educational benefit is minimal after a few attempts. To ensure that the modules provide a meaningful educational experience, we are implementing the concept of mastery. A certain level of proficiency in the material, not a perfect score, should be the students' goal when running these modules. Once the student demonstrates the specified level of proficiency, he/she has the option to exit the module, and any grade higher than the mastery level is considered equivalent to mastery. We have found in testing the modules this semester that this small change has kept the focus on the concepts rather than the score. 
Table 3 Current Status of Interactive Computer Modules Project

\begin{tabular}{|c|c|c|}
\hline Course & Module & Status \\
\hline \multirow[t]{6}{*}{ Introduction } & UNITS-Unit conversion & Disseminated \\
\hline & GAS--Equations of state & Disseminated \\
\hline & SODA POP_Vapor-liquid equilibrium & Disseminated \\
\hline & HAWAII-Material balances & Disseminated \\
\hline & BEER-Material and energy balances & Disseminated \\
\hline & LPCVD—Stoichiometry tables & In progress \\
\hline \multirow[t]{5}{*}{ Fluid Mechanics } & BRIDGE*_-Shell balances & Student tested \\
\hline & SHOOT*_Simplifying equations of change & Student tested \\
\hline & DIMNO--Dimensionless groups & In progress \\
\hline & VISC_Fluid viscosity & Student tested \\
\hline & PIPING-Piping networks & In progress \\
\hline \multirow[t]{4}{*}{ Transport } & BRIDGE*_-Shell balances & Student tested \\
\hline & SHOOT*-Simplifying equations of change & Student tested \\
\hline & DRUG PATCH-Diffusion & Student tested \\
\hline & MACRO-Macroscopic balances & Student tested \\
\hline \multirow[t]{5}{*}{ Separations } & ISEP-Bases of separation & Student tested \\
\hline & McCABE-McCabe-Thiele distillation & Student tested \\
\hline & CASCADES—Cascades of equilibrium stages & Student tested \\
\hline & ABSORPTION_Absorption processes & Student tested \\
\hline & RESCUE-Adsorption & Student tested \\
\hline \multirow[t]{8}{*}{ Reaction Kinetics } & KINETIC JEOPARDY_Mole balances & Student tested \\
\hline & STAGE—Reactor staging & Student tested \\
\hline & KINETIC JEOPARDY 2-Rate laws & Student tested \\
\hline & TIC-TAC-TOE-Isothermal reactor design & Student tested \\
\hline & SWAMP_Analysis of rate data & In progress \\
\hline & HET CAT_Rate laws and mechanism & In progress \\
\hline & INDY - Nonisothermal reactor simulation & In progress \\
\hline & LPCVD_-Mass transfer effects in CVD & In progress \\
\hline
\end{tabular}

* BRIDGE and SHOOT are applicable to both Fluid Mechanics and Transport courses.

\section{CURRENT STATUS}

We have made great progress toward our goal of five to eight interactive computer modules per course. Table 3 includes a description of the modules developed so far. Except for the LPCVD module, the introductory course modules have already been disseminated to approximately 40 departments around the country. The fluids/transport, separations, and kinetics modules that have been student tested have also been tested by professors at other universities, with very positive results. Modules that are still in progress are expected to be completed by the end of this summer.

\section{FUTURE PLANS}

We have developed a large number of useful interactive computer modules for the chemical engineering curriculum. This summer we will employ seven undergraduate students, one masters student, and an additional staff member (a recent graduate of our department) to produce new modules and complete the existing modules. The latter two people worked with the project last summer. The focus will be on increasing the coverage within the courses already addressed, and to upgrade the introductory course modules by introducing many of the techniques we have learned during this project. We also plan to work on a new module on problem-solving techniques, most likely focusing on the Kepner-Tregoe strategies [3]. In addition, we are considering updating an existing BASICA module that demonstrates interactively how to critique journal articles. The purpose of this exercise is to develop skills on how to read the literature with a critical eye and to suggest new experiments or theories in order to make definitive conclusions. Finally, we will develop distribution and installation materials in preparation for the transfer of the module distribution management to the CACHE corporation. 


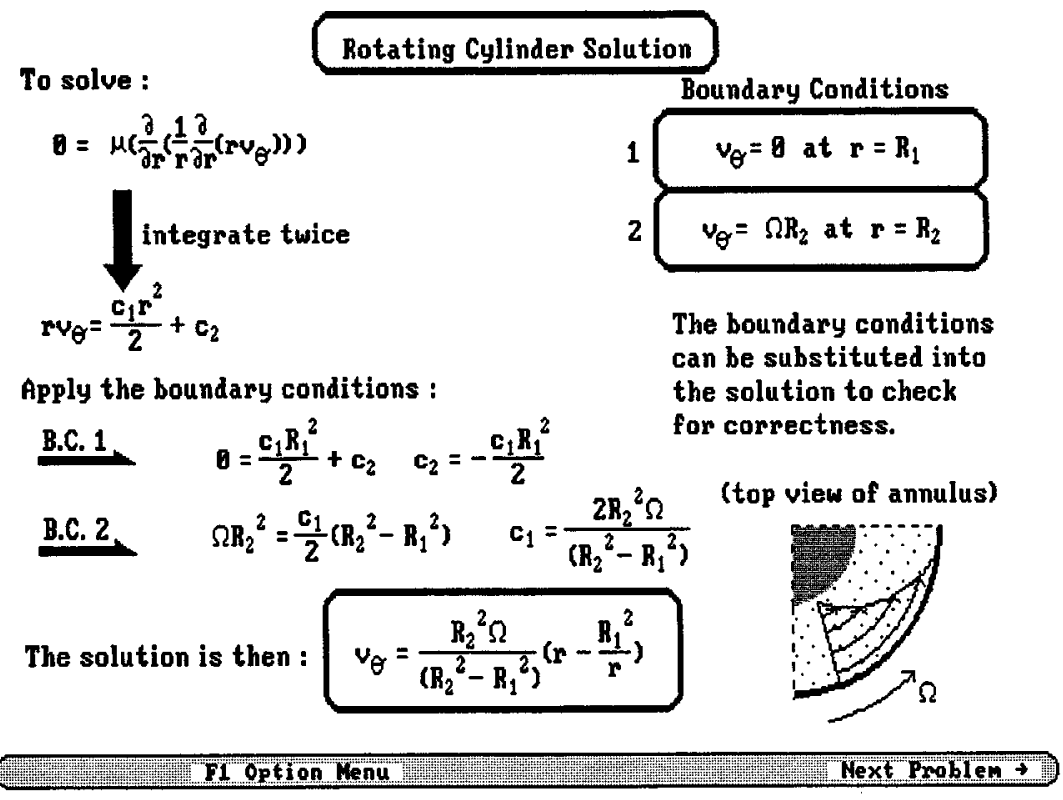

Figure 16 Solution Screen, "Shoot," Transport Equations of Change Module.

\section{DESCRIPTION OF MODULES ENCLOSED}

Some of the modules we are developing are included in this issue. These modules have been mentioned in the earlier discussion, but they are described individually and in more detail in this section.

\section{Shooting Gallery}

In the simplification of the equations of change module, applicable to both the fluid mechanics and transport courses, the student may first review the equations of change and run through a short demonstration of the interactive screens. The student is then presented with three of eight possible momentum or energy balance problems, such as the one shown in Figure 11, and must simplify the equations of change for each problem to obtain the velocity profile within the system for the momentum balance problems, or the temperature profile for the energy balance ones. This is done by "shooting down" the terms that can be eliminated. If the student chooses to "shoot down" a term, the module provides all the reasons why the term could have been eliminated, or, if the term should not have been eliminated, the reasons the term must remain in the equation. Guidance is also provided in the form of the overlay screen shown in Figure 15. After the student completes the simplification, the resulting equations are solved to yield the temperature or ve- locity profile for the system, as shown in Figure 16, for the problem described in Figure 11.

\section{McCabe-Thiele}

In the distillation module for the separations course, after a brief review and quiz of distillation processes, the student is presented with a desired separation. The student is allowed to vary three of five process parameters in a column (distillate flow rate, bottoms mole fraction, condition of feed stream, reflux ratio, and boilup ratio) to affect the required separation. The effect of parameter variations on the performance of the distillation column is shown on a McCabe-Thiele diagram, as in Figure 5. In this way the student is allowed to explore and learn from experience the effect of process parameters on the performance of the distillation column.

\section{Tic-Tac-Toe}

The optional review section in this module on isothermal reactor design allows the student to review the basic equations that govern the performance of an isothermal reactor. Nine simulations covering various aspects of isothermal reactor design are then available to the student in the format of a Tic-TacToe game, shown in Figure 12. The student must correctly master the concepts covered in enough of the simulations to "win" the game. Each simulation consists of an explanation of the current reactor and 
operating conditions (Figure 13), a simulation in which the student is allowed to explore the effect of one of the parameters on the reactor conditions (such as in Figure 14), and three follow-up question to test that the student has mastered the concepts covered by the simulation.

\section{SUMMARY}

Interactive computer modules can provide an added dimension to the teaching of engineering concepts. They provide students with additional exposure to the material presented in class, and allow them to study the application of the concepts to actual processes; explore the effect of variation of parameters on the system; and, in general, test their understanding of the material. Some of the issues of importance to interactive computer module developers, such as techniques to eliminate tediousness, prevent guessing, keep focus on the concepts, introduce new technologies, and provide individual guidance to the student, were discussed.

\section{ACKNOWLEDGMENTS}

The authors thank the faculty of the Chemical Engineering Department at the University of Michigan for their assis-

\section{BIOGRAPHIES}

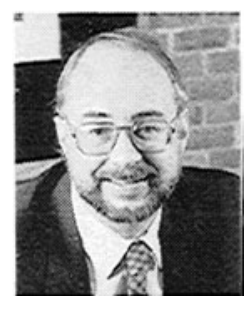

H. Scott Fogler is the Ame and Catherine Vennema Professor of Chemical Engineering at the University of Michigan. He has been involved in the development of individualized learning materials since 1974 . He is the author of over 100 research papers, and his recent textbook, Elements of Chemical Reaction Engineering, is currently used by the majority of chemical engineering departments in the United States.

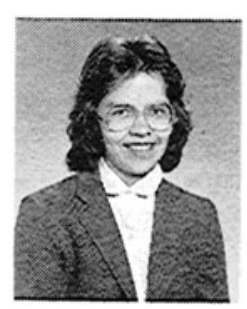

Susan M. Montgomery, a research fellow at the University of Michigan Department of Chemical Engineering, is project manager for the interactive computer modules project. She received a BSE in Chemical Engineering from the University of Michigan and an MS and $\mathrm{PhD}$ from Princeton University. tance as technical advisors, and for allowing the modules to be tested in the undergraduate courses. The assistance of Professors Michael Cutlip (University of Connecticut), Sangtae Kim (University of Wisconsin), and J. D. Seader (University of Utah) as technical advisors is also gratefully acknowledged, as are the contributions of Dr. Samer Naser, who served as supervisor for many of the modules for the separations course. Financial support from the National Science Foundation grant "A Focus on Developing Innovative Engineers" as well as the generous support of the College of Engineering is much appreciated. Finally, many thanks to the many undergraduate programmers who have been involved in this project over the years, making this project possible.

\section{REFERENCES}

[1] C. C. Kulik and J. A. Kulik, AEDS J. Vol. 19, p. 81, 1986.

[2] J. A. Kulik and C. C. Kulik, Contemp. Educational Philos., Vol. 12, p. 222, 1987.

[3] C. H. Kepner and B. B. Tregoe, The New Rational Manager. Princeton University Press, Princeton, NJ, 1981.

Robert P. Zipp was the previous project manager for the interactive computer modules project. He received a BS and an MS in Chemical Engineering from the University of Missouri-Rolla, and a PhD from the University of Arizona. He is currently employed at the Upjohn Co., Kalamazoo, Michigan. (Photo not available at this time.) 